\title{
Н.К. Смоленцев
}

\section{О ПОЧТИ (ПАРА) КОМПЛЕКСНЫХ СТРУКТУРАХ КЭЛИ НА СФЕРАХ $\mathrm{S}^{2,4}$ И $\mathrm{S}^{3,3}$}

\begin{abstract}
Изучаются почти комплексные и почти пара-комплексные структуры Кэли на шестимерных псевдоримановых сферах в пространстве чисто мнимых октав расщепляемой алгебры Кэли $\mathbf{C a}^{\prime}$. Показано, что структуры Кэли неинтегрируемы, вычислены их основные геометрические характеристики. В отличие от обычной римановой сферы $\mathbf{S}^{6}$, на рассматриваемых псевдосферах существуют (интегрируемые) комплексные структуры пара-комплексные структуры.
\end{abstract}

Ключевые слова: алгебра Кэли, расщепляемая алгебра Кэли, группа $G_{2}$, сплит-октонионы, векторное произведение, почти комплексная структура, почти пара-комплексная структура, шестимерные псевдоримановы сферы.

\section{1. Введение}

Почти комплексные структуры на обычной шестимерной сфере $\mathbf{S}^{6}$ изучаются давно и активно, (см., например, библиографию и исторический обзор по этой теме в статьях [1 и 2]). В 1958 г. LeBrun показал, что ортогональные почти комплексные структуры $J$ на $\mathbf{S}^{6}$ не интегрируемы, а в 1999 г. Bor и HernandezLamoneda показали, что неинтегрируемыми будут почти комплексные структуры $J$, ортогональные не только относительно стандартной метрики $g_{0}$, но и для метрик, близких к стандартной. Однако вопрос о существовании на $\mathbf{S}^{6}$ интегрируемых почти комплексных структур не решен до сих пор. Среди ортогональных почти комплексных структур $J$ на $\left(\mathbf{S}^{6}, g_{0}\right)$ особое место занимает почти комплексная структура Кэли $J_{0}$, которая получается при помощи векторного произведения в объемлющем пространстве $\mathbf{R}^{7}$ чисто мнимых октав Кэли. Структура Кэли $J_{0}$ является инвариантной относительно действия компактной особой группы $G_{2}$, при этом $\mathrm{S}^{6} \cong G_{2} / S U(3)$. В работе [3] для структуры Кэли $J_{0}$ на $\mathbf{S}^{6}$ получены аналитические выражения фундаментальной формы и ее внешнего дифференциала через калибровки пространства $\mathbf{R}^{7}$, найден тензор Нейенхейса через тройное векторное произведение и показано, что фундаментальная 2-форма $\omega$ является собственной для оператора Лапласа. Классификация инвариантных почти комплексных структур на однородных многообразиях размерности 6 с полупростой группой изотропии представлена в работе [4].

Как известно [5], существует расщепляемая алгебра Кэли $\mathbf{C a}^{\prime}$, которая получается из кватернионов процедурой удвоения Кэли - Диксона с использованием «мнимого» числа $\boldsymbol{e}$, такого, что $\boldsymbol{e}^{2}=+1$. Алгебра Кэли Са' $\mathbf{a}^{\prime}$ имеет псевдоевклидово скалярное произведение сигнатуры $(4,4)$, определенное квадратичной формой $|x \bar{x}|^{2}=x_{0}^{2}+x_{1}^{2}+x_{2}^{2}+x_{3}^{2}-x_{4}^{2}-x_{5}^{2}-x_{6}^{2}-x_{7}^{2}$. Группой автоморфизмов алгебры $\mathbf{C a}^{\prime}$ 
является некомпактная особая группа $G_{2}^{*}$. Пространство чисто мнимых сплитоктонионов наследует квадратичную форму $g$ сигнатуры $(3,4)$ и является поэтому псевдоевклидовым пространством $\mathbf{R}^{3,4}$. Существуют два типа сфер в пространстве $\mathbf{R}^{3,4}$ : действительного и мнимого радиуса. Псевдосфера $\mathbf{S}^{2,4}$ действительного радиуса является однородным псевдоримановым многообразием $\mathrm{S}^{2,4} \cong G_{2}^{*} / S U(1,2)$ сигнатуры $(2,4)$. Псевдосфера $\mathbf{S}^{3,3}(i)$ мнимого радиуса является однородным псевдоримановым многообразием $\mathrm{S}^{3,3} \cong G_{2}^{*} / S L(3, \mathrm{R})$ сигнатуры $(3,3)$.

Умножение в алгебре $\mathbf{C} \mathbf{a}^{\prime}$ определяет в пространстве $\mathbf{R}^{3,4}$ чисто мнимых октав векторное произведение, инвариантное относительно $G_{2}^{*}$. Это позволяет определить на сфере $\mathbf{S}^{2,4} \subset \mathbf{R}^{3,4}$ ортогональную почти комплексную структуру Кэли $J$ через умножение касательных векторов на вектор нормали.

В данной работе показано, что такая структура не интегрируема и вычислен ее тензор Нейенхейса через тройное векторное произведение. Найдено выражение фундаментальной 2-формы $\omega$ почти эрмитовой структуры на $\mathbf{S}^{2,4}$ и показано, что 2-форма $\omega$ является собственной для оператора Лапласа. В отличие от обычной римановой сферы $\mathbf{S}^{6}$, на $\mathbf{S}^{2,4}$ существуют интегрируемые почти комплексные структуры.

На сфере $\mathbf{S}^{3,3}(i) \subset \mathbf{R}^{3,4}$ мнимого радиуса векторное произведение в $\mathbf{R}^{3,4}$ определяет почти пара-комплексную структуру $P$. Показано, что она не интегрируема, вычислен тензор Нейенхейса через тройное векторное произведение. Найдено выражение фундаментальной 2-формы $\omega$ на $\mathbf{S}^{3,3}(i)$ и показано, что $\omega$ является собственной 2-формой оператора Лапласа. В отличие от обычной римановой сферы $\mathbf{S}^{6}$, на $\mathbf{S}^{3,3}(i)$ существуют интегрируемые почти пара-комплексные структуры, которые легко строятся с использованием стереографической проекции.

\section{2. Предварительные сведения}

\section{1. Алгебры Кэли}

Пусть Н - алгебра кватернионов $w=x^{0}+x^{1} \boldsymbol{e}_{1}+x^{2} \boldsymbol{e}_{2}+x^{3} \boldsymbol{e}_{3}$. Алгебра Са чисел Кэли получается процедурой удвоения Кэли - Диксона. Для этого вводится еще одна мнимая единица $\boldsymbol{e}$ и образуются числа Кэли в виде $x=a+b \boldsymbol{e}$, где $a$ и $b-$ кватернионы. Умножение таких чисел определяется по формуле $x y=(a+b e)(c+d e)=(a c-\bar{d} b)+(d a+b \bar{c}) e$. Группой автоморфизмов алгебры Кэли Са является простая особая компактная группа $G_{2}$.

Известен другой вариант процедуры удвоения Кэли - Диксона. В этом случае дополнительная «единица» $\boldsymbol{e}$ обладает свойством $\boldsymbol{e}^{2}=+1$. Тогда мы получаем так называемые сплит-октонионы (split-octonions) в виде $x=a+b \boldsymbol{e}$, где $a$ и $b$ - кватернионы. Умножение таких чисел определяется по формуле

$$
x y=(a+b e)(c+d e)=(a c+\bar{d} b)+(d a+b \bar{c}) e .
$$

В результате получается неассоциативная алгебра $\mathbf{C a}^{\prime}$, которая называется расщепляемой (split) алгеброй Кэли. В дальнейшем удобно ввести обозначения: $\boldsymbol{e}_{4}=\boldsymbol{e}$, $\boldsymbol{e}_{5}=\boldsymbol{e}_{1} \boldsymbol{e}, \boldsymbol{e}_{6}=\boldsymbol{e}_{2} \boldsymbol{e}, \boldsymbol{e}_{7}=\boldsymbol{e}_{3} \boldsymbol{e}$. Отметим, что $\boldsymbol{e}_{i}^{2}=-1$ при $i=1,2,3$ и $\boldsymbol{e}_{j}^{2}=+1$ при $j=4$, $5,6,7$. Каждое сплит-число Кэли записывается в виде $x=x^{0}+x^{1} \boldsymbol{e}_{1}+\ldots+x^{7} \boldsymbol{e}_{7}$, 
где $x_{\alpha} \in \mathbf{R}$, а числа $\boldsymbol{e}_{1}, \boldsymbol{e}_{2}, \ldots, \boldsymbol{e}_{7}-$ мнимые единицы. Имеют место следующие правила их перемножения (первый сомножитель в столбце слева, а второй - в строке сверху):

\begin{tabular}{|c|c|c|c|c|c|c|c|}
\hline & $\boldsymbol{e}_{1}$ & $\boldsymbol{e}_{2}$ & $\boldsymbol{e}_{3}$ & $\boldsymbol{e}_{4}$ & $\boldsymbol{e}_{5}$ & $\boldsymbol{e}_{6}$ & $\boldsymbol{e}_{7}$ \\
\hline $\boldsymbol{e}_{1}$ & -1 & $\boldsymbol{e}_{3}$ & $-\boldsymbol{e}_{2}$ & $\boldsymbol{e}_{5}$ & $-\boldsymbol{e}_{4}$ & $-\boldsymbol{e}_{7}$ & $\boldsymbol{e}_{6}$ \\
\hline $\boldsymbol{e}_{2}$ & $-\boldsymbol{e}_{3}$ & -1 & $\boldsymbol{e}_{1}$ & $\boldsymbol{e}_{6}$ & $\boldsymbol{e}_{7}$ & $-\boldsymbol{e}_{4}$ & $-\boldsymbol{e}_{5}$ \\
\hline $\boldsymbol{e}_{3}$ & $\boldsymbol{e}_{2}$ & $-\boldsymbol{e}_{1}$ & -1 & $\boldsymbol{e}_{7}$ & $-\boldsymbol{e}_{6}$ & $\boldsymbol{e}_{5}$ & $-\boldsymbol{e}_{4}$ \\
\hline $\boldsymbol{e}_{4}$ & $-\boldsymbol{e}_{5}$ & $-\boldsymbol{e}_{6}$ & $-\boldsymbol{e}_{7}$ & +1 & $-\boldsymbol{e}_{1}$ & $-\boldsymbol{e}_{2}$ & $-\boldsymbol{e}_{3}$ \\
\hline $\boldsymbol{e}_{5}$ & $\boldsymbol{e}_{4}$ & $-\boldsymbol{e}_{7}$ & $\boldsymbol{e}_{6}$ & $\boldsymbol{e}_{1}$ & +1 & $\boldsymbol{e}_{3}$ & $-\boldsymbol{e}_{2}$ \\
\hline $\boldsymbol{e}_{6}$ & $\boldsymbol{e}_{7}$ & $\boldsymbol{e}_{4}$ & $-\boldsymbol{e}_{5}$ & $\boldsymbol{e}_{2}$ & $-\boldsymbol{e}_{3}$ & +1 & $\boldsymbol{e}_{1}$ \\
\hline $\boldsymbol{e}_{7}$ & $-\boldsymbol{e}_{6}$ & $\boldsymbol{e}_{5}$ & $\boldsymbol{e}_{4}$ & $\boldsymbol{e}_{3}$ & $\boldsymbol{e}_{2}$ & $-\boldsymbol{e}_{1}$ & +1 \\
\hline
\end{tabular}

Напомним основные свойства алгебры $\mathbf{C a}^{\prime}$, подробнее об этом см. [5]. Сопряжение сплит-октонионов задается обычным образом, $\bar{x}=x_{0}-x_{1} \boldsymbol{e}_{1}-\ldots-x_{7} \boldsymbol{e}_{7}$, и обладает свойством $\overline{x y}=\bar{y} \bar{x}$. Алгебра Кэли Са' имеет квадратичную форму $N(x)=x \bar{x}=x_{0}^{2}+x_{1}^{2}+x_{2}^{2}+x_{3}^{2}-x_{4}^{2}-x_{5}^{2}-x_{6}^{2}-x_{7}^{2}$ и соответствующее псевдоевклидово скалярное произведение $\langle x, y\rangle=(x \bar{y}+y \bar{x}) / 2$ сигнатуры $(4,4)$. Поэтому алгебру $\mathbf{C a}^{\prime}$ мы будем часто рассматривать как псевдоевклидово пространство $\mathbf{R}^{4,4}$. Алгебра $\mathbf{C a}^{\prime}$ является композиционной [5], поскольку выполняется равенство $N(x y)=N(x) N(y)$.

Алгебра $\mathbf{C a}^{\prime}$ неассоциативна, т.е. $(x y) z \neq x(y z)$. Ассоциатором называется выражение $[x, y, z]=(x y) z-x(y z)$. Расщепленная алгебра $\mathbf{C a}^{\prime}$ является альтернативной, т.е. выполняются свойства

- $(x x) y=x(x y), x(y y)=(x y) y, \forall x, y \in \mathbf{C a}^{\prime}$.

Это название такие алгебры получили потому, что ассоциатор кососимметричен (альтернативен) по всем аргументам. В частности, свойство альтернативности записывается через ассоциатор следующим образом: $[x, x, y]=0,[x, y, y]=0$. Отметим еще ряд свойств алгебры $\mathbf{C a}^{\prime}$ :

- $x(y x)=(x y) x$

- $(x \bar{x}) y=x(\bar{x} y), x(\bar{y} y)=(x \bar{y}) y$, т.е. $[x, \bar{x}, y]=0$ и $[x, \bar{y}, y]=0$;

- $(x x y) x=x x(y x)$, т.e. $[x x, y, x]=0$;

- $((x y) z) y=x(y z y), \quad(x y x) z=x(y(x z)), \quad(x y)(z x)=x(y z) x$.

\section{2. Группа $\boldsymbol{G}_{\mathbf{2}}{ }^{*}$}

Комплексная исключительная простая группа Ли $\boldsymbol{G}_{2}$ имеет две вещественных формы. Одна из них, компактная, обозначается символами $G_{2}{ }^{c}$ или $G_{2}$ и хорошо известна [6]. Вторая, некомпактная, вещественная форма обозначается $G_{2}{ }^{*}$, ее описание можно найти в [7]. Эта группа $G_{2}{ }^{*}$ может быть определена как группа автоморфизмов расщепляемой алгебры $\mathbf{C a}^{\prime}$. Поэтому $G_{2}{ }^{*} \subset S O(4,3)$. Группу $G_{2}{ }^{*}$ можно также понимать, как стабилизатор векторного произведения на семимерном псевдоевклидовом пространстве $V$ сигнатуры $(3,4)$. Выберем в псевдоевклидовом пространстве $V$ базис $\left(e_{1}, e_{2}, \ldots, e_{7}\right)$, в котором квадратичная форма принимает вид $g=-2\left(e^{1} \cdot e^{5}+e^{2} \cdot e^{6}+e^{3} \cdot e^{7}\right)-\left(e^{4}\right)^{2}$. Тогда группа $G_{2}{ }^{*}$ является стабилизатором следующей 3-формы на $V: \Omega_{0}=\sqrt{2}\left(e^{123}-e^{567}\right)+e^{4} \wedge\left(e^{15}+e^{26}+e^{37}\right)$. 


\section{3. Векторное произведение}

Векторным ( $r$-местным) произведением на вещественном $n$-мерном векторном пространстве $V$ с невырожденной билинейной формой $\langle x, y\rangle$ называется [8] полилинейное отображение $P: V^{r} \rightarrow V(1 \leq r \leq n)$, которое обладает свойствами

$$
\left\langle P\left(x_{1}, \ldots, x_{r}\right), x_{i}\right\rangle=0, \quad 1 \leq i \leq r \quad \text { и } \quad\left\|P\left(x_{1}, \ldots, x_{r}\right)\right\|^{2}=\operatorname{det}\left(\left\langle x_{i}, x_{j}\right\rangle\right) .
$$

Одноместные векторные произведения - это ортогональные комплексные структуры $J$ на четномерном векторном пространстве $V$. Двухместное векторное произведение существует только в размерности 3 и 7. На семимерном векторном пространстве $V$ они описываются следующим образом [8]. Пусть $W$ - композиционная алгебра и $V$ - ортогональное дополнение к единице $\boldsymbol{e}$ в алгебре $W$. Определим $P: V \times V \rightarrow V$ формулой $P(x, y)=x y+\langle x, y\rangle$ e. Тогда $P$ является двухместным векторным произведением и, наоборот, каждое такое векторное произведение возникает таким образом. Если $\operatorname{dim} W=8$, мы получаем 2-местное векторное произведение на 7-мерном пространстве $V$. Билинейная форма, ассоциированная с таким векторным произведением, имеет сигнатуру $(0,7)$ или $(4,3)$. Группа автоморфизмов есть либо $G_{2}$ (компактная форма), либо $G_{2}{ }^{*}$ (некомпактная форма). Любые два двуместных векторных произведения являются изоморфными.

Трехместные векторные произведения. Пусть $V$ - композиционная алгебра и $\langle x, y\rangle$ - соответствующая билинейная форма. Определим $P_{1}, P_{2}: V \times V \rightarrow V$ формулами [8]:

$$
\begin{aligned}
& P_{1}(x, y, z)=\varepsilon(-x(\bar{y} z)+<x, y>z+<y, z>x-<z, x>y), \\
& P_{2}(x, y, z)=\varepsilon(-(x \bar{y}) z+<x, y>z+<y, z>x-<z, x>y),
\end{aligned}
$$

где $\varepsilon= \pm 1$. Тогда $P_{1}$ и $P_{2}$ представляют собой трехместные векторные произведения на $V$ относительно билинейной формы $\varepsilon\langle x, y\rangle$ и обратно каждое трехместное векторное произведение возникает таким образом.

Алгебра Кэли $\mathbf{C a}^{\prime}$ является композиционной с билинейной формой сигнатуры $(4,4)$, поэтому на ней определены трехместные векторные произведения $P_{1}$ и $P_{2}$. Операция сопряжения определяет антиизоморфизм этих векторных произведений. Поэтому в дальнейшем мы будем рассматривать на $\mathbf{C a}^{\prime}$ только второе векторное произведение $P$ :

$$
P(x, y, z)=-(x \bar{y}) z+<x, y>z+<y, z>x-<z, x>y .
$$

Отметим, что если векторы $x, y, z$ взаимно ортогональны, то $P(x, y, z)=-(x \bar{y}) z$.

\section{4. Псевдосферы в пространстве $\mathbf{R}^{3,4}$}

Рассмотрим семимерное пространство $\mathbf{R}^{7}$ чисто мнимых элементов $X=x_{1} \boldsymbol{e}_{1}$ $+\ldots+x_{7} \boldsymbol{e}_{7}$ расщепляемой алгебры $\mathbf{C a}^{\prime}$. Оно наследует квадратичную форму $g$ сигнатуры $(3,4)$ и является поэтому псевдоевклидовым пространством $\mathbf{R}^{3,4}$. В пространстве $\mathbf{R}^{3,4}$ можно определить два вида (псевдо)сфер:

$$
\begin{gathered}
x_{1}^{2}+x_{2}^{2}+x_{3}^{2}-x_{4}^{2}-x_{5}^{2}-x_{6}^{2}-x_{7}^{2}=r^{2} \\
x_{1}^{2}+x_{2}^{2}+x_{3}^{2}-x_{4}^{2}-x_{5}^{2}-x_{6}^{2}-x_{7}^{2}=-r^{2} .
\end{gathered}
$$


Первая псевдосфера $\mathbf{S}^{2,4}(r)$ имеет действительный радиус $r>0$, она пересекает оси $O x_{1}, O x_{2}, O x_{3}$. Касательные плоскости являются псевдоевклидовыми сигнатуры $(2,4)$. Скалярные квадраты векторов нормалей к $\mathbf{S}^{2,4}(r)$ положительны, а их квадраты в алгебре Кэли $\mathbf{C a}^{\prime}$ - отрицательны. Группа $G_{2}{ }^{*}$ действует транзитивно и изометрично на $\mathbf{S}^{2,4}(r)$, а ее группа изотропии совпадает с $S U(1,2)$. Поэтому сфера $\mathbf{S}^{2,4}(r)$ является однородным пространством: $S^{2,4} \cong G_{2}^{*} / S U(1,2)$. Отметим также, что $\mathrm{S}^{2,4} \cong S O(3,4) / S O(2,4)$.

Вторая псевдосфера $\mathbf{S}^{3,3}(\mathrm{ir})$ мнимого радиуса $i r$, она пересекает оси $O x_{4}, \ldots$, $O x_{7}$. Касательные плоскости являются псевдоевклидовыми сигнатуры $(3,3)$. Скалярные квадраты векторов нормалей к $\mathbf{S}^{3,3}(i r)$ отрицательны, а их квадраты в алгебре Кэли $\mathbf{C a}^{\prime}$ - положительны. Группа $G_{2}{ }^{*}$ действует транзитивно и изометрично на $\mathbf{S}^{3,3}(\mathrm{ir})$, а ее группа изотропии совпадает с $S L(3, \mathbf{R})$. Поэтому сфера $\mathbf{S}^{3,3}(\mathrm{ir})$ является однородным пространством: $\mathrm{S}^{3,3} \cong G_{2}^{*} / S L(3, \mathrm{R})$.

\section{5. Почти пара-комплексные структуры}

Почти пара-комплексной структурой на $2 n$-мерном многообразии $M$ называется [9] поле $P$ эндоморфизмов касательного расслоения $T M$, таких, что $P^{2}=I d$ и ранги собственных распределений $T^{ \pm} M:=\operatorname{ker}(I d \mp P)$ равны. Почти паракомплексная структура $P$ называется интегрируемой, если распределения $T^{\Perp} M$ инволютивны. В этом случае $P$ называется пара-комплексной структурой. Тензор Нейенхейса $N_{P}$ почти пара-комплексной структуры $P$ определяется равенством

$$
N_{P}(X, Y)=2([X, Y]+[P X, P Y]-P[P X, Y]-P[X, P Y])
$$

для всех векторных полей $X, Y$ на $M$. Как и в комплексном случае, паракомплексная структура $P$ интегрируема тогда и только тогда, когда $N_{P}=0$. В работе [9] представлен обзор теории и подробно рассмотрены инвариантные паракомплексные и пара-кэлеровы структуры на группах Ли.

\section{3. Векторное произведение в $\mathrm{R}^{3,4}=\operatorname{Im}\left(\mathrm{Ca}^{\prime}\right)$}

Пусть $\mathbf{C a}^{\prime}$ - расщепляемая алгебра Кэли. Как обычно, числа вида $x=x^{0}$ будем называть действительными, а числа $X=x^{1} \boldsymbol{e}_{1}+x^{2} \boldsymbol{e}_{2}+\ldots+x^{7} \boldsymbol{e}_{7}$ - чисто мнимыми. Будем записывать сплит-октонионы в виде суммы $x=x^{0}+X$. Пространство $\mathbf{R}^{3,4}=\operatorname{Im}\left(\mathbf{C a}^{\prime}\right) \quad$ мнимых $\quad$ октав наследует из $\mathbf{C a}^{\prime}$ скалярное произведение $g(X, Y)=\langle X, Y\rangle$ сигнатуры $(3,4)$. Пусть $x=X, y=Y$ - два чисто мнимых сплитоктониона. Определим векторное произведение элементов $X, Y \in \operatorname{Im}\left(\mathbf{C a}^{\prime}\right)$ как мнимую часть их произведения в алгебре $\mathbf{C a}$ :

$$
X \times Y=\operatorname{Im}(X Y) \text {. }
$$

Легко видеть, что

$$
X \times Y=X Y+g(X, Y) .
$$

Также легко видеть, что данное векторное произведение $X \times Y$ билинейно, кососимметрично и ортогонально каждому их сомножителей. Умножение в алгебре $\mathbf{C a}^{\prime}$ выражается через векторное произведение следующим образом: для $x=x^{0}+X$ и $y=y^{0}+Y$ имеем $x y=\left(\begin{array}{ll}x_{0} & y_{0}-\langle X, Y\rangle\end{array}\right)+x_{0} Y+y_{0} X+X \times Y$. Для любых векторов $X, Y \in \operatorname{Im}\left(\mathbf{C a}^{\prime}\right)$ имеет место следующее равенство:

$$
X \times(X \times Y)=-g(X, X) Y+g(X, Y) X .
$$


В частности, если $\boldsymbol{n}$ - вектор единичной длины, то для любого $Y \in \mathbf{R}^{3,4}$ выполняется равенство

$$
\boldsymbol{n} \times(\boldsymbol{n} \times Y)=-Y+g(\boldsymbol{n}, Y) \boldsymbol{n} .
$$

Смешанное произведение определяется равенством $(X Y Z)=g(X, Y \times Z)=$ $=g(X \times Y, Z)$ и представляет собой кососимметричную 3-форму $\Omega$ на $\mathbf{R}^{3,4}$,

$$
\Omega(X, Y, Z)=g(X \times Y, Z) .
$$

В обозначениях $\omega^{p q r}=d x^{p} \wedge d x^{q} \wedge d x^{r} 3$-форма $\Omega$ имеет следующее выражение:

$$
\Omega=\omega^{123}-\omega^{145}+\omega^{167}-\omega^{246}-\omega^{257}-\omega^{347}+\omega^{356} .
$$

Поскольку на $\mathbf{R}^{3,4}$ имеется псевдориманова метрика и соответствующая форма объема $\mu=\sqrt{|\operatorname{det}(g)|} d x^{1} \wedge \cdots \wedge d x^{7}=\omega^{1234567}$, то определен оператора Ходжа $*: \Lambda^{k}\left(\mathbf{R}^{3,4}\right) \rightarrow \Lambda^{7-k}\left(\mathbf{R}^{3,4}\right)$. Поэтому на пространстве $\mathbf{R}^{3,4}=\operatorname{Im}\left(\mathbf{C a}^{\prime}\right)$ определена внешняя 4-форма $\Psi=* \Omega$,

$$
\Psi=\omega^{4567}-\omega^{2367}+\omega^{2345}-\omega^{1357}-\omega^{1346}-\omega^{1256}+\omega^{1247} .
$$

Очевидно, что $\Omega=* \Psi$. Вычисляя значения $\Psi$ на векторах базиса $\boldsymbol{e}_{1}, \ldots, \boldsymbol{e}_{7}$, легко видеть, что имеет место следующее выражение на векторах $X, Y, Z, W \in \mathbf{R}^{3,4}$ :

$$
\Psi(X, Y, Z, W)=g(X,(Y \times Z) \times W)=-g(X, Y \times(Z \times W)) .
$$

Лемма 1. На пространстве $\mathbf{R}^{3,4}=\operatorname{Im}\left(\mathbf{C a}^{\prime}\right)$ мнимых октав ассоциатор $[X, Y, Z]$ может быть выражен следующей формулой:

$$
[X, Y, Z]=2(X \times Y) \times Z+2 g(Y, Z) X-2 g(Z, X) Y .
$$

Доказательство. Используя кососимметричность 3-формы $\Omega$ и ассоциатора $[X, Y, Z]$, а также формулу $X Y=X \times Y-g(X, Y)$, получаем

$$
\begin{gathered}
{[X, Y, Z]=(X Y) Z-X(Y Z)=(X \times Y) \times Z-X \times(Y \times Z)-g(X \times Y, Z)+g(X, Y \times Z)-} \\
-g(X, Y) Z+g(Y, Z) X==(X \times Y) \times Z-X \times(Y \times Z)-g(X, Y) Z+X \cdot g(Y, Z) .
\end{gathered}
$$

Теперь используем это выражение в следующей сумме и получаем необходимую формулу:

$$
[X, Y, Z]=[X, Y, Z]+[Z, X, Y]+[X, Z, Y]=2(X \times Y) \times Z+2 g(Y, Z) X-2 g(Z, X) Y .
$$

Следствие. Имеет место следующая формула:

$$
(X \times Y) \times Z-g(X, Z) Y+g(Y, Z) X=-X \times(Y \times Z)+g(X, Z) Y-g(X, Y) Z .
$$

Доказательство. Следует из равенства $[X, Y, Z]=-[Z, Y, X]$.

Нам потребуются также свойства векторного произведения, которые сразу следуют из предыдущего равенства:

- Если $\boldsymbol{n}, Y, Z \in \mathbf{R}^{3,4}$ и если $Y, Z \perp \boldsymbol{n}$, то

$$
(\boldsymbol{n} \times Y) \times Z=-\boldsymbol{n} \times(Y \times Z)-g(Y, Z) \boldsymbol{n} .
$$

- Если $\boldsymbol{n}$ - вектор единичной длины, то для любых $Y, Z \in \mathbf{R}^{3,4}$ $\boldsymbol{n} \times(\boldsymbol{n} \times Z)=-Z+g(\boldsymbol{n}, Z) \boldsymbol{n}, \quad g(\boldsymbol{n} \times Y, \boldsymbol{n} \times Z)=g(Y, Z)-g(Y, \boldsymbol{n}) g(Z, \boldsymbol{n})$.

- $\quad$ Если же $g(\boldsymbol{n}, \boldsymbol{n})=-1$, то для любых $Y, Z \in \mathbf{R}^{3,4}$

$$
\boldsymbol{n} \times(\boldsymbol{n} \times Z)=Z+g(\boldsymbol{n}, Z) \boldsymbol{n}, \quad g(\boldsymbol{n} \times Y, \boldsymbol{n} \times Z)=-g(Y, Z)-g(Y, \boldsymbol{n}) g(Z, \boldsymbol{n}) .
$$




\section{4. Почти комплексная структура на $S^{2,4}(r)$}

Рассмотрим сферу $\mathbf{S}^{2,4}=\mathbf{S}^{2,4}(1)$ единичного радиуса в пространстве $\mathbf{R}^{3,4}$ $=\operatorname{Im}\left(\mathbf{C a}^{\prime}\right)$ мнимых сплит-октав Кэли. Касательные плоскости $T_{x} \mathbf{S}^{2,4}$ к сфере являются псевдоевклидовыми сигнатуры $(2,4)$. Скалярные квадраты векторов нормалей $\boldsymbol{n}(x)=x$ к сфере $\mathbf{S}^{2,4}$ положительны. Рассмотрим векторное умножение касательных векторов $Y \in T_{x} \mathbf{S}^{2,4}$ на вектор нормали $\boldsymbol{n}(x)=x$. Легко видеть, что эта операция переводит касательное пространство в себя: если $Y \in T_{x} \mathbf{S}^{2,4}$, т.е., $g(Y, \boldsymbol{n})=0$, то

$$
g(\boldsymbol{n} \times Y, \boldsymbol{n})=0, \quad \text { т.е. } \boldsymbol{n} \times Y \in T_{x} \mathbf{S}^{2,4} .
$$

Из свойства векторного произведения следует

$$
\boldsymbol{n} \times(\boldsymbol{n} \times Y)=-g(\boldsymbol{n}, \boldsymbol{n}) Y+g(\boldsymbol{n}, Y) \boldsymbol{n}=-Y .
$$

Это означает, что оператор $J_{x}(Y)=\boldsymbol{n} \times Y$ левого умножения касательных векторов в точке $x \in \mathbf{S}^{2,4}$ на вектор нормали $\boldsymbol{n}(x)=x$ к сфере $\mathbf{S}^{2,4}$ определяет на $T_{x} \mathbf{S}^{2,4}$ комплексную структуру. Поскольку такая операция определена в каждой точке $x \in$ $\mathbf{S}^{2,4}$, то мы получаем, что единичная сфера $\mathbf{S}^{2,4}$ имеет естественную почти комплексную структуру $J$, которую мы будем называть структурой Кэли:

$$
J_{x}: T_{x} \mathbf{S}^{2,4} \rightarrow T_{x} \mathbf{S}^{2,4}, \quad J_{x}(Y)=\boldsymbol{n}(x) \times Y .
$$

Из равенства $g(\boldsymbol{n} \times X, \boldsymbol{n} \times Y)=g(X, Y)-g(X, \boldsymbol{n}) g(Y, \boldsymbol{n})$ сразу следует ее ортогональность.

Пусть $\omega(X, Y)=g(J X, Y)$ - фундаментальная 2-форма, соответствующая $J$. Поскольку $g(X \times Y, Z)=\Omega(X, Y, Z)$, то легко видеть, что

$$
\omega(X, Y)=g(\boldsymbol{n} \times X, Y)=\Omega(\boldsymbol{n}, X, Y)=\Omega(X, Y, \boldsymbol{n})=g(X \times Y, \boldsymbol{n})=g(\boldsymbol{n}, X \times Y) .
$$

Лемма 2. Форма $\Omega$ при ее ограничении на сферу обладает свойством

$$
\Omega(J X, Y, Z)=\Omega(X, J Y, Z)=\Omega(X, Y, J Z) .
$$

Доказательство. Используем формулу $(\boldsymbol{n} \times Y) \times Z=-\boldsymbol{n} \times(Y \times Z)-g(Y, Z) \boldsymbol{n}$ и равенство $g(X, Y \times Z)=g(X \times Y, Z)$ :

$$
\begin{gathered}
\Omega(Z, J X, Y)=g(Z, J X \times Y)=g(Z,(\boldsymbol{n} \times X) \times Y)=g(Z,-\boldsymbol{n} \times(X \times Y)-g(X, Y) \boldsymbol{n})=g(Z,- \\
\boldsymbol{n} \times(X \times Y))=-g(Z \times \boldsymbol{n}, X \times Y)=g(\boldsymbol{n} \times Z, X \times Y)=g(J Z, X \times Y)=\Omega(J Z, X, Y) .
\end{gathered}
$$

Лемма 3. Для любых $X, Y, Z \in T_{x} \mathbf{S}^{2,4}$ имеет место равенство

$$
i_{n} \Psi(X, Y, Z)=-\Omega(J X, Y, Z),
$$

где $\boldsymbol{i}_{\boldsymbol{n}}$ - внутреннее произведение с вектором нормали $\boldsymbol{n}(x)$.

Доказательство. $\boldsymbol{i}_{\boldsymbol{n}} \Psi(X, Y, Z)=-g(\boldsymbol{n}, X \times(Y \times Z))=-g(\boldsymbol{n} \times X, Y \times Z)=-\Omega(J X, Y, Z)$.

Пусть $\boldsymbol{e}_{1}, \ldots, \boldsymbol{e}_{6}$ - ортонормированный базис пространства $T_{x} \mathbf{S}^{2,4}$, в котором $\boldsymbol{e}_{1}$ и $\boldsymbol{e}_{2}$ являются пространственноподобными, а остальные - времениподобны. Тогда $\boldsymbol{e}_{0}=\boldsymbol{n}(x), \boldsymbol{e}_{1}, \ldots, \boldsymbol{e}_{6}-$ ортонормированный базис пространства $\mathbf{R}^{3,4}=\mathbf{R} x \oplus T_{x} \mathbf{S}^{2,4}$. Пусть $\mu=\boldsymbol{e}^{0} \wedge \boldsymbol{e}^{1} \wedge \ldots \wedge \boldsymbol{e}^{6}$ - элемент объема $\mathbf{R}^{3,4}$ и $\mu_{\mathrm{S}}=\boldsymbol{e}^{1} \wedge \ldots \wedge \boldsymbol{e}^{6}$ - элемент объема сферы $\mathbf{S}^{2,4}$ (в точке $x$ ). Очевидно, $\boldsymbol{\mu}_{\mathrm{S}}=\boldsymbol{i}_{\boldsymbol{n}} \boldsymbol{\mu}$, где $\boldsymbol{i}_{\boldsymbol{n}}-$ внутреннее произведение с вектором нормали $\boldsymbol{n}(x)$. Пусть $*_{\mathrm{S}}$ и $*_{\mathrm{R}}$ - операторы Ходжа на сфере и на $\mathbf{R}^{3,4}$ соответственно. Символом $\left.\theta\right|_{\mathrm{S}}$ будем обозначать ограничение дифференциальной формы $\theta$ в $\mathbf{R}^{3,4}$ на подмногообразие $\mathbf{S}^{2,4}$, а символом $\left.\theta\right|_{n}-$ нормальную компоненту формы $\theta$ вдоль $\mathbf{S}^{2,4}$, то есть, $\left.\theta\right|_{n}=\boldsymbol{n} \wedge \tilde{\theta}$.

Лемма 4. Пусть $\theta-k$-форма на $\mathbf{R}^{3,4}$ и $\left.\theta\right|_{\mathrm{S}}$ - ее ограничение на касательное пространство $T_{x} \mathbf{S}^{2,4} \subset \mathbf{R}^{3,4}$. Тогда для операторов Ходжа $*_{\mathrm{S}} u *_{\mathrm{R}}$ на сфере и на $\mathbf{R}^{3,4}$ соответственно выполняются равенства

$$
*_{\mathrm{R}} \theta=(-1)^{k} \boldsymbol{n} \wedge *_{\mathrm{S}} \theta, \quad *_{\mathrm{S}}\left(\left.\theta\right|_{\mathrm{S}}\right)=(-1)^{k} \boldsymbol{i}_{\boldsymbol{n}}\left(*_{\mathrm{R}} \theta\right) .
$$


Доказательство. Форму $\theta$ можно записать в виде $\theta=\left.\theta\right|_{S}+\boldsymbol{n} \wedge \tilde{\theta}$, где $\tilde{\theta}-$ форма степени $k-1$, которая выражается через $\boldsymbol{e}^{1}, \ldots, \boldsymbol{e}^{6}$. Очевидно, что $i_{\boldsymbol{n}}\left(*_{\mathrm{R}}(\boldsymbol{n} \wedge \tilde{\theta})\right)=0$. Поэтому достаточно доказать лемму для $\theta=\left.\theta\right|_{\mathrm{S}}$. Для любой $k$-формы $v$ на $\mathbf{S}^{2,4}$ имеем $v \wedge *_{\mathrm{S}} \theta=g(v, \theta) \mu_{\mathrm{S}}$ Из этого равенства, с учетом того, что $\boldsymbol{\mu}=\boldsymbol{n} \wedge \mu_{\mathrm{S}}, \quad$ получаем $\boldsymbol{n} \wedge \nu \wedge *_{\mathrm{S}} \theta=g(\nu, \theta) \boldsymbol{n} \wedge \mu_{\mathrm{S}}=g(\nu, \theta) \mu$ или, что то же самое, $(-1)^{k} \vee \wedge \boldsymbol{n} \wedge *_{\mathrm{s}} \theta=g(v, \theta) \mu=v \wedge *_{\mathrm{R}} \theta$. Последнее равенство верно не только для форм $v$ на $T_{x} \mathbf{S}^{2,4}$, но и для любых $k$-форм $v=\left.v\right|_{S}+\boldsymbol{n} \wedge \tilde{v}$ на $\mathbf{R}^{7}$. На второй компоненте $\boldsymbol{n} \wedge \tilde{\boldsymbol{v}}$ обе части равенства обращаются в нуль. Поэтому мы получаем равенство $(-1)^{k} \boldsymbol{n} \wedge *_{\mathrm{S}} \theta=*_{\mathrm{R}} \theta$. Применяя внутреннее произведение $\boldsymbol{i}_{\boldsymbol{n}}$ к обеим частям последнего равенства, получаем утверждение леммы.

Теорема 1. Фундаментальная форма $\omega$ почти комплексной структуры Кэли J на $\mathbf{S}^{2,4}$ и ее внешний дифференциал d

$$
\begin{gathered}
\omega=\boldsymbol{i}_{n} \Omega, \quad d \omega=\left.3 \Omega\right|_{\mathrm{S}}, \\
*_{\mathrm{S}} \omega=\left.\Psi\right|_{\mathrm{S}}, \quad{ }_{\mathrm{S}} d \omega=-3 \boldsymbol{i}_{\boldsymbol{n}} \Psi, \\
d \omega(X, Y, Z)=3 \boldsymbol{i}_{\boldsymbol{n}} \Psi(J X, Y, Z), \\
d \omega(J X, Y, Z)=d \omega(X, J Y, Z)=d \omega(X, Y, J Z), \\
d \omega(X, J Y, J Z)=-d \omega(X, Y, Z),
\end{gathered}
$$$$
\omega \wedge d \omega=0 .
$$

Доказательство. Первое равенство вытекает из определения $\omega$ и $\Omega$ : $\boldsymbol{i}_{\boldsymbol{n}} \Omega(X, Y)=g(\boldsymbol{n}, X \times Y)=\omega(X, Y)$ для любых $X, Y$, касательных к сфере. Во втором равенстве мы считаем, что векторное поле $\boldsymbol{n}(x)=x$ определено на всем $\mathbf{R}^{7}$. Тогда, поскольку 3-форма $\Omega$ с постоянными коэффициентами в $\mathbf{R}^{7}$, является замкнутой, поэтому $d \boldsymbol{i}_{n} \Omega=L_{n} \Omega=3 \Omega$, где $L_{\boldsymbol{n}}=d \cdot \boldsymbol{i}_{\boldsymbol{n}}+\boldsymbol{i}_{\boldsymbol{n}} \cdot d-$ производная Ли вдоль векторного поля $\boldsymbol{n}(x)=x$ на $\mathbf{R}^{7}$. Следовательно, $d \omega=d \boldsymbol{i}_{n} \Omega=L_{n} \Omega=3 \Omega$. Вторая пара равенств получается из лемм 2 и 3 и из первых свойств, $*_{\mathrm{S}} \omega=\left.*_{\mathrm{S}}\left(\boldsymbol{i}_{\boldsymbol{n}} \Omega\right)\right|_{\mathrm{S}}=\boldsymbol{i}_{\boldsymbol{n}} *_{\mathrm{R}}\left(i_{\boldsymbol{n}} \Omega\right)$. Подробнее, пусть $\mathbf{e}_{\boldsymbol{n}}$ - оператор внешнего умножения на 1-форму, дуальную векторному полю $\boldsymbol{n}(x)=x$. Как известно, на $k$-формах $v$ операторы $\boldsymbol{i}_{\boldsymbol{n}}$ и $\mathbf{e}_{\boldsymbol{n}}$ связаны соотношением $\boldsymbol{i}_{\boldsymbol{n}} *_{\mathrm{R}}=(-1)^{k} *_{\mathrm{R}} \mathbf{e}_{\boldsymbol{n}}$. Поэтому

$$
*_{\mathrm{S}} \omega=\boldsymbol{i}_{\boldsymbol{n}} *_{\mathrm{R}}\left(i_{\boldsymbol{n}} \Omega\right)=*_{\mathrm{R}} \mathbf{e}_{\boldsymbol{n}}\left(i_{\boldsymbol{n}} \Omega\right)=*_{\mathrm{R}}\left(\left.\Omega\right|_{n}\right)=\left.\Psi\right|_{\mathrm{S}} .
$$

Далее, $*_{S} d \omega=\left.3 *_{S} \Omega\right|_{S}=-3 \boldsymbol{i}_{\boldsymbol{n}} *_{\mathrm{R}} \Omega=-3 \boldsymbol{i}_{\boldsymbol{n}} \Psi$. Равенство (3) вытекает из (1) и из леммы 2. Свойства (4), (5) следуют из (1) и леммы 1. Последнее равенство следует из (1) и из $i_{n}(\Omega \wedge \Omega)=0$.

Замечание. Фундаментальная 2-форма $\omega$ на $\mathbf{S}^{2,4}$ является козамкнутой $\delta \omega=0$. Действительно, 4-форма $\Psi$ на $\mathbf{R}^{7}$ имеет постоянные коэффициенты, поэтому $d \Psi=0$. Хорошо известно, что на $k$-формах кодифференциал $\delta$ имеет выражение: $\delta=(-1)^{k} *^{-1} d *=(-1)^{k n+n+1+\eta} * d *$, где $\eta-$ число «минусов» метрического тензора $g$. В нашем случае $\eta=4$ и $n=6$. Следовательно, $\delta=-* d *$. Поэтому

$$
\delta \omega=-* d * \omega=-* d\left(\left.\Psi\right|_{\mathrm{S}}\right)=-\left.*(d \Psi)\right|_{\mathrm{S}}=0 .
$$

В работе [10] Хитчин определил понятие невырожденности (стабильности) для 3-форм $\Phi$ на шестимерном вещественном векторном пространстве $V$ и построил для 3-формы Ф линейный оператор $K_{\Phi}$, квадрат которого пропорционален тождественному оператору: $K_{\Phi}{ }^{2}=\lambda(\Phi) I d$. Если $\lambda(\Phi)<0$, то $K_{\Phi}$ определяет на $V$ комплексную структуру, а если $\lambda(\Phi)>0$, то пара-комплексную структуру. Пусть $\mu$ 
- форма объема на $V$. Оператор $K_{\Phi}$ определяется следующей формулой: $\mathbf{1}_{K_{\Phi}(X)} \mu=\imath_{X} \Phi \wedge \Phi$.

Для фундаментальной формы $\omega$ почти комплексной структуры Кэли на $\mathbf{S}^{2,4} 3$ форма $d \omega$ имеет вид $d \omega=\left.3 \Omega\right|_{\mathrm{S}}$, где $\Omega=\omega^{123}-\omega^{145}+\omega^{167}-\omega^{246}-\omega^{257}-\omega^{347}+\omega^{356}$. Найдем оператор Хитчина [5] $K_{d \omega}$ для 3-формы $d \omega$ на сфере $\mathbf{S}^{2,4}$. Рассмотрим сначала точку $x=e_{1} \in \mathbf{S}^{2,4}$. Касательное пространство $T_{x} \mathbf{S}^{2,4}$ имеет ортонормированный базис векторов $\boldsymbol{e}_{2}, \ldots, \boldsymbol{e}_{7}$ и форму объема $\mu=\omega^{234567} .2$-форма $d \omega$ на $T_{x} \mathbf{S}^{2,4}$ имеет вид

$$
d \omega_{x}=3\left(-\omega^{246}-\omega^{257}-\omega^{347}+\omega^{356}\right) .
$$

2-форма $d \omega_{x}$ является инвариантной при действии на $T_{x} \mathbf{S}^{2,4}$ подгруппы изотропии $S U(1,2) \subset G_{2}{ }^{*}$.

Поэтому достаточно вычислить $K_{d \omega}$ на одном векторе, например $K_{d \omega}\left(\boldsymbol{e}_{2}\right)$ :

$$
\begin{aligned}
& \mathrm{l}_{e_{2}} d \omega_{x} \wedge d \omega_{x}=9\left(-\omega^{46}-\omega^{57}\right)\left(-\omega^{246}-\omega^{257}-\omega^{347}+\omega^{356}\right)= \\
& =9\left(\omega^{46} \wedge \omega^{257}+\omega^{57} \wedge \omega^{246}\right)=-18 \omega^{24567}=18 \mathrm{i}_{e_{3}} \omega^{234567}=18 \mathrm{i}_{e_{3}} \mu .
\end{aligned}
$$

Поэтому $K_{d \omega}\left(\boldsymbol{e}_{2}\right)=18 \boldsymbol{e}_{3}=18 \boldsymbol{e}_{1} \times \boldsymbol{e}_{2}=18 J\left(\boldsymbol{e}_{2}\right)$. Отсюда следует, что $I_{d \omega}=J_{x}$. Из инвариантности почти комплексной структуры Кэли $J$ и формы $\Omega$ относительно группы $G_{2}{ }^{*}$, действующей транзитивно на $\mathbf{S}^{2,4}$, мы получаем, что равенство $I_{d \omega}=J_{x}$ имеет место во всех точках псевдосферы $\mathbf{S}^{2,4}$.

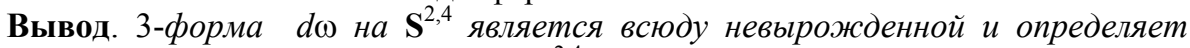
почти комплексную структуру $I_{d \omega} н а \mathbf{S}^{2,4}$, которая совпадает с почти комплексной структурой Кэли J.

Вычислим тензор Нейенхейса $N(X, Y)=2([J X, J Y]-[X, Y]-J[J X, Y]-J[X, J Y])$ почти комплексной структуры $J[11]$. Для этого найдем ковариантную производную тензора $J$. Поскольку $\boldsymbol{n}(x)=x$, то для любого касательного вектора $X \in T_{x} \mathbf{S}^{2,4}$ имеем $D \boldsymbol{n}_{x}(X)=X$. Будем считать, что касательные векторы $X, Y \in T_{x} \mathbf{S}^{2,4}$ продолжены на пространство $\mathbf{R}^{7}$ как постоянные векторные поля и $\boldsymbol{n}(x)=x$ также определено на $\mathbf{R}^{7} \backslash\{0\}$. Тогда имеют место равенства $\left(\nabla_{X} J\right) Y=\operatorname{pr}_{x}\left(D_{X}(J Y)\right)=$ $=\operatorname{pr}_{x}\left(D_{X}(\boldsymbol{n} \times Y)\right)=\operatorname{pr}_{x}(X \times Y)$, где $\mathrm{pr}-$ ортогональная в $\mathbf{R}^{3,4}$ проекция на касательное пространство $T_{x} \mathbf{S}^{2,4}, \operatorname{pr}_{x}(Z)=Z-g(Z, \boldsymbol{n}) \boldsymbol{n}$. Получаем

$$
\left(\nabla_{X} J\right) Y=X \times Y-g(\boldsymbol{n}, X \times Y) \boldsymbol{n}=X \times Y-\omega(X, Y) \boldsymbol{n} .
$$

Замечание. Из полученного выражения $\nabla_{X} J$ сразу вытекает кососимметричность оператора $\nabla_{X} J$ и, в частности, свойство $\nabla_{\mathrm{X}}(J) X=0$ приближенной псевдокэлеровости многообразия $\mathbf{S}^{2,4}$ и равенство $\nabla_{J X} J=-J \nabla_{X} J$.

Теорема 2. Тензор Нейенхейса $N(X, Y)$ почти комплексной структуры Кэли $J$ на $\mathbf{S}^{2,4}$ имеет вид

$$
N(X, Y)=-8 \boldsymbol{n} \times(X \times Y) .
$$

Доказательство. В книге [11] приведена формула

$$
4 g\left(\left(\nabla_{\mathrm{Z}} J\right) X, Y\right)=6 \boldsymbol{d} \Phi(Z, J X, J Y)-6 \boldsymbol{d} \Phi(Z, X, Y)+g(N(X, Y), J Z),
$$

где $\Phi(X, Y)=g(X, J Y)$ - фундаментальная форма. В нашем случае фундаментальная форма $\omega(X, Y)=g(J X, Y)=-\Phi(X, Y)$. Кроме того, в [6] берется другое определение внешнего умножения. Поэтому формула для внешнего дифференциала тоже меняется. Внешний дифференциал $\boldsymbol{d}$ в [11] берется с коэффициентом $1 / 3: \boldsymbol{d} \omega=d \omega / 3$. Учитывая это, имеем в нашем случае $4 g\left(\left(\nabla_{Z} J\right) X, Y\right)=-2 d \omega(Z, J X, J Y)+2 d \omega(Z, X, Y)+$ $g(N(X, Y), J Z)$, поэтому $g(N(X, Y), J Z)=4 g\left(\left(\nabla_{Z} J\right) X, Y\right)+2 d \omega(Z, J X, J Y)-2 d \omega(Z, X, Y)$. Tогда, учитывая, что $d \omega(X, Y, Z)=3 g(X, Y \times Z)$, имеем 


$$
\begin{gathered}
g(N(X, Y), J Z)=4 g\left(\left(\nabla_{Z} J\right) X, Y\right)+2 d \omega(Z, J X, J Y)-2 d \omega(Z, X, Y)= \\
=4 g(Z \times X-2 \omega(Z, X) \boldsymbol{n}, Y)-2 d \omega(X, Y, Z)-2 d \omega(Z, X, Y)= \\
=4 g(Z \times X, Y)-12 g(X, Y \times Z)=4 g(Z, X \times Y)-12 g(X \times Y, Z)= \\
=-8 g(\boldsymbol{n} \times(X \times Y), \boldsymbol{n} \times Z)=-8 g(\boldsymbol{n} \times(X \times Y), J Z) .
\end{gathered}
$$

Теорема 3. Фундаментальная 2-форма $\omega$ почти комплексной структуры Кэли на $\mathbf{S}^{2,4}$ является собственной для оператора Лапласа: $\Delta \omega=12 \omega$.

Доказательство. Лапласиан, действующий на дифференциальных формах, определяется формулой $\Delta=d \delta+\delta d$, где $\delta-$ кодифференциал. Хорошо известно, что на $k$-формах кодифференциал $\delta$ имеет выражение $\delta=(-1)^{k} *^{-1} d *=$ $=(-1)^{k n+n+1+\eta} * d *$, где $\eta-$ число «минусов» метрического тензора $g$. В нашем случае $\eta=4$ и $n=6$. Следовательно, $\delta=-* d *$. Для формы $\omega$ известно, что $\delta \omega=0$. Поэтому достаточно вычислить $\delta d \omega$. Будем обозначать внешний дифференциал в пространстве $\mathbf{R}^{7}$ с индексом $d_{\mathrm{R}}$. Используем свойство $*_{\mathrm{S}}\left(\left.\theta\right|_{\mathrm{S}}\right)=(-1)^{k} i_{\boldsymbol{n}}\left(*_{\mathrm{R}} \theta\right)$, установленное в лемме 4 , и результаты теоремы 1 :

$$
\begin{gathered}
\delta d \omega=-*_{\mathrm{S}} d *_{\mathrm{S}}(d \omega)=-3 *_{\mathrm{S}} d *_{\mathrm{S}}\left(\left.\Omega\right|_{\mathrm{S}}\right)=-3(-1)^{3} *_{\mathrm{S}} d\left(\boldsymbol{i}_{\boldsymbol{n}} *_{\mathrm{R}} \Omega\right)= \\
=\left.3 *_{\mathrm{S}}\left(d_{\mathrm{R}} \boldsymbol{i}_{\boldsymbol{n}}(\Psi)\right)\right|_{\mathrm{S}}=\left.3 *_{\mathrm{S}}\left(L_{n} \Psi\right)\right|_{\mathrm{S}}=12 *_{\mathrm{S}}\left(\left.\Psi\right|_{\mathrm{S}}\right)=12 \boldsymbol{i}_{\boldsymbol{n}} *_{\mathrm{R}} \Psi=12 \boldsymbol{i}_{\boldsymbol{n}} \Omega=12 \omega .
\end{gathered}
$$

В этих вычислениях мы использовали производную Ли $L_{n}=d \cdot \boldsymbol{i}_{\boldsymbol{n}}+\boldsymbol{i}_{\boldsymbol{n}} \cdot \boldsymbol{d}$ вдоль векторного поля $\boldsymbol{n}(x)=x$ на $\mathbf{R}^{7} \backslash\{0\}$. 4-форма $\Psi$ с постоянными коэффициентами в $\mathbf{R}^{7}$ является замкнутой, поэтому $d \boldsymbol{i}_{\boldsymbol{n}} \Psi=L_{\boldsymbol{n}} \Psi$. Кроме того, поле $\boldsymbol{n}(x)=x$ задает растяжение $\mathbf{R}^{7} \backslash\{0\}$, следовательно, для 4-формы $\Psi$ с постоянными коэффициентами выполняется равенство $L_{n} \Psi=4 \Psi$. Теорема доказана.

Комплексная структура на $S^{2,4}(r)$. В этом пункте мы покажем, что на псевдосфере $\mathbf{S}^{2,4}$ существуют интегрируемые комплексные структуры. Рассмотрим стереографическую проекцию $\mathbf{S}^{2,4}$ из точки начала координат $\mathbf{R}^{7}$ на часть цилиндра $\mathbf{S}^{2} \times D^{4}$, где $\mathbf{S}^{2}-$ стандартная единичная сфера в пространстве $\mathbf{R}^{3}$ с координатами $\left(x_{1}, x_{2}, x_{3}\right)$ и $D^{4}$ - единичный шар в пространстве $\mathbf{R}^{4}$ с координатами $\left(x_{4}, x_{5}, x_{6}, x_{7}\right)$, $x_{4}^{2}+x_{5}^{2}+x_{6}^{2}+x_{7}^{2}<1$. Прямая, проходящая через начало координат и точку $x=\left(x_{1}, x_{2}, \ldots, x_{7}\right)$ псевдосферы $x_{1}^{2}+x_{2}^{2}+x_{3}^{2}-x_{4}^{2}-x_{5}^{2}-x_{6}^{2}-x_{7}^{2}=1$, пресекает цилиндр $\mathbf{S}^{2} \times D^{4}$ в точке $y$ с координатами

$$
y_{i}=\frac{x_{i}}{\sqrt{x_{1}^{2}+x_{2}^{2}+x_{3}^{2}}}, \quad i=1,2, \ldots, 7 .
$$

Ясно, что первые переменные $\left(y_{1}, y_{2}, y_{3}\right)$ описывают двумерную сферу $\mathbf{S}^{2}$ в $\mathbf{R}^{3}$. Остальные координаты меняются в единичном шаре $D^{4}$ в пространстве $\mathbf{R}^{4}$. Действительно, пусть $r^{2}=x_{1}^{2}+x_{2}^{2}+x_{3}^{2}$. На псевдосфере $r^{2} \geq 1$. Тогда из уравнения псевдосферы следует, что

$$
\frac{x_{4}^{2}+x_{5}^{2}+x_{6}^{2}+x_{7}^{2}}{x_{1}^{2}+x_{2}^{2}+x_{3}^{2}}=1-\frac{1}{x_{1}^{2}+x_{2}^{2}+x_{3}^{2}}, \text { или } 0 \leq y_{4}^{2}+y_{5}^{2}+y_{6}^{2}+y_{7}^{2}=1-\frac{1}{r^{2}}<1 .
$$

Через каждую точку $\left(y_{4}, y_{5}, y_{6}, y_{7}\right) \in D^{4}$, при фиксированной точке $\left(y_{1}, y_{2}, y_{3}\right) \in \mathbf{S}^{2}$, проходит единственная прямая, пересекающая псевдосферу в точке $x_{i}=r \cdot y_{i}$, $i=1,2, \ldots, 7$, при $r=1 / \sqrt{1-y_{4}^{2}-y_{5}^{2}-y_{6}^{2}-y_{7}^{2}}$ и, наоборот, каждая прямая, проходящая через начало координат и точку $x \in \mathbf{S}^{2,4}$, пересекает шар $D^{4}$ при определенных $\left(y_{1}, y_{2}, y_{3}\right) \in \mathbf{S}^{2}$. 


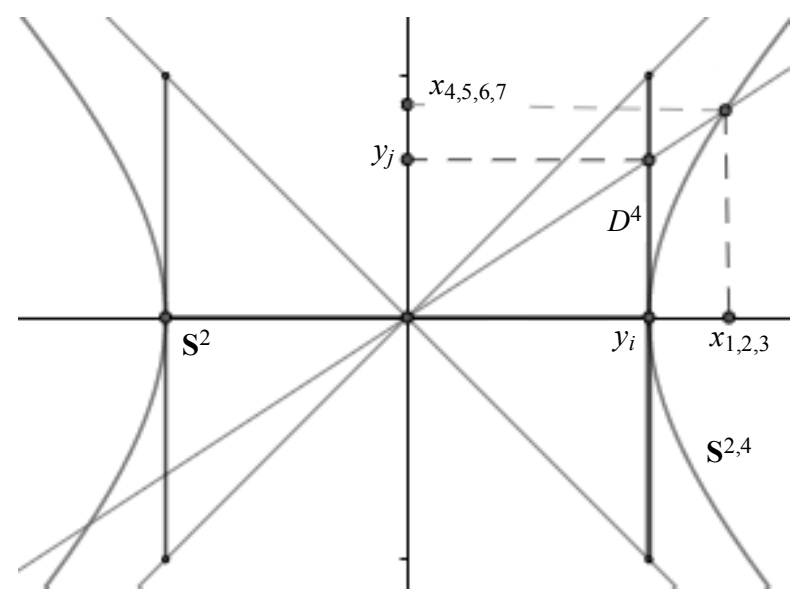

Рис. 1. Стереографическая проекция

Fig. 1. Stereographic projection

Поскольку прямое произведение $\mathbf{S}^{2} \times D^{4}$ допускает комплексные структуры, то диффеоморфизм стереографической проекции $f: \mathbf{S}^{2,4} \rightarrow \mathbf{S}^{2} \times D^{4}$ позволяет перенести комплексные структуры с $\mathbf{S}^{2} \times D^{4}$ на сферу $\mathbf{S}^{2,4}$. Отметим, что по построению $\mathbf{S}^{2} \times D^{4}$ является псевдоримановым сигнатуры $(2,4)$.

\section{5. Почти пара-комплексная структура на $S^{3,3}(i)$}

Результаты этой части аналогичны тем, что были получены для сферы $\mathbf{S}^{2,4}$, однако имеются и некоторые отличия.

Рассмотрим сферу $\mathbf{S}^{3,3}=\mathbf{S}^{3,3}(i)$ чисто мнимого единичного радиуса в пространстве $\mathbf{R}^{3,4}=\operatorname{Im}\left(\mathbf{C a}^{\prime}\right)$ мнимых сплит-октав Кэли. Касательные плоскости к сфере $T_{x} \mathbf{S}^{3,3}, \forall x \in \mathbf{S}^{3,3}$, являются псевдоевклидовыми сигнатуры $(3,3)$. Скалярные квадраты векторов нормалей $\boldsymbol{n}(x)=x$ к сфере $\mathbf{S}^{3,3}$ отрицательны, $g(\boldsymbol{n}, \boldsymbol{n})=-1$.

Векторное умножение касательных векторов $Y \in T_{x} \mathbf{S}^{3,3}$ на вектор нормали $\boldsymbol{n}(x)=x$ переводит касательное пространство в себя: если $Y \in T_{x} \mathbf{S}^{3,3}$, то есть, $g(Y, \boldsymbol{n})=0$, то

$$
g(\boldsymbol{n} \times Y, \boldsymbol{n})=0 \text {, т.е. } \boldsymbol{n} \times Y \in T_{x} \mathbf{S}^{3,3} .
$$

Из свойства векторного произведения следует, что

$$
\boldsymbol{n} \times(\boldsymbol{n} \times Y)=-g(\boldsymbol{n}, \boldsymbol{n}) Y+g(\boldsymbol{n}, Y) \boldsymbol{n}=Y .
$$

Это означает, что $\left(P_{x}\right)^{2}=I d$. Оператор $P_{x}(X)=\boldsymbol{n} \times X$ кососимметричен, $g(\boldsymbol{n} \times X, Y)=$ $=-g(X, \boldsymbol{n} \times Y)$, но не ортогонален: $g\left(P_{x} X, P_{x} Y\right)=g(\boldsymbol{n} \times X, \boldsymbol{n} \times Y)=-g(X, Y), \forall X, Y \in T_{x} \mathbf{S}^{3,3}$. Оператор $P_{x}$ пространственноподобные векторы переводит во времениподобные векторы. Собственные подпространства оператора $P_{x}$ являются трехмерными изотропными подпространствами, касательными к $\mathbf{S}^{3,3}$. Поэтому оператор $P_{x}(Y)=\boldsymbol{n} \times Y$ умножения касательных векторов на вектор нормали $\boldsymbol{n}(x)=x$ к сфере $\mathbf{S}^{3,3}$ определяет на $T_{x} \mathbf{S}^{3,3}$ пара-комплексную структуру. Такая операция определена в каждой точке $x \in \mathbf{S}^{3,3}$, следовательно, мы получаем, что единичная псевдориманова сфера $\mathbf{S}^{3,3}$ имеет естественную почти пара-комплексную структуру $P$, которую мы будем называть почти пара-комплексной структурой Кэли:

$$
P_{x}: T_{x} \mathbf{S}^{3,3} \rightarrow T_{x} \mathbf{S}^{3,3}, \quad P_{x}(Y)=\boldsymbol{n}(x) \times Y, \quad \forall x \in \mathbf{S}^{3,3}, \forall Y \in T_{x} \mathbf{S}^{3,3} .
$$


Пусть $\omega(X, Y)=g(P X, Y)-$ фундаментальная 2-форма, соответствующая $P$, и пусть $\Omega(X, Y, Z)=g(X \times Y, Z)$. Тогда легко видеть, что

$$
\omega(X, Y)=g(\boldsymbol{n} \times X, Y)=\Omega(\boldsymbol{n}, X, Y)=\Omega(X, Y, \boldsymbol{n})=g(X \times Y, \boldsymbol{n})=g(\boldsymbol{n}, X \times Y) .
$$

Напомним, что на пространстве $\mathbf{R}^{3,4}=\operatorname{Im}\left(\mathbf{C a}^{\prime}\right)$ определена внешняя 4-форма $\Psi=* \Omega$.

Пусть $*_{\mathrm{S}}$ и $*_{\mathrm{R}}$ - операторы Ходжа на сфере и на $\mathbf{R}^{3,4}$ соответственно. Символом $\left.\theta\right|_{\mathrm{S}}$ будем обозначать ограничение дифференциальной формы $\theta$ в $\mathbf{R}^{3,4}$ на подмногообразие $\mathbf{S}^{3,3}$, а символом $\left.\theta\right|_{n}-$ нормальную компоненту формы $\theta$ вдоль $\mathbf{S}^{3,3}$, то есть, $\left.\theta\right|_{n}=\boldsymbol{n} \wedge \tilde{\theta}$.

Для почти пара-комплексной структуры Кэли совершенно аналогично доказываются все результаты, установленные в разделе 3.2 для почти комплексных структур Кэли:

1. Форма $\Omega$ при ее ограничении на сферу обладает свойством

$$
\Omega(P X, Y, Z)=\Omega(X, P Y, Z)=\Omega(X, Y, P Z) .
$$

2. Для любых $X, Y, Z \in T_{x} \mathbf{S}^{3,3}$ имеет место равенство

$$
\boldsymbol{i}_{\boldsymbol{n}} \Psi(X, Y, Z)=-\Omega(P X, Y, Z) .
$$

3. Пусть $\theta$ - $k$-форма на $\mathbf{R}^{3,4}$ и $\left.\theta\right|_{\mathrm{S}}$ - ее ограничение на касательное пространство $T_{x} \mathbf{S}^{3,3} \subset \mathbf{R}^{3,4}$. Тогда

$$
*_{\mathrm{R}} \theta=(-1)^{k} \boldsymbol{n} \wedge *_{\mathrm{S}} \theta, \quad *_{\mathrm{S}}\left(\left.\theta\right|_{\mathrm{S}}\right)=(-1)^{k} \boldsymbol{i}_{\boldsymbol{n}}\left(*_{\mathrm{R}} \theta\right) .
$$

4. Фундаментальная форма $\omega$ почти пара-комплексной структуры Кэли Р на $\mathbf{S}^{3,3}$ и ее внешний дифференциал

$$
\begin{gathered}
\omega=\boldsymbol{i}_{\boldsymbol{n}} \Omega, \quad d \omega=\left.3 \Omega\right|_{\mathrm{S}}, \quad *_{\mathrm{S}} \omega=\left.\Psi\right|_{\mathrm{S}}, \quad *_{\mathrm{S}} d \omega=-3 \boldsymbol{i}_{\boldsymbol{n}} \Psi, \quad \omega \wedge d \omega=0, \\
d \omega(X, Y, Z)=3 \boldsymbol{i}_{\boldsymbol{n}} \Psi(P X, Y, Z), \quad d \omega(X, Y, Z)=3 g(X, Y \times Z), \\
d \omega(P X, Y, Z)=d \omega(X, P Y, Z)=d \omega(X, Y, P Z), d \omega(X, P Y, P Z)=-d \omega(X, Y, Z) .
\end{gathered}
$$

Для фундаментальной формы $\omega$ почти пара-комплексной структуры Кэли на $\mathbf{S}^{3,3}$ 3-форма $d \omega$ имеет вид $d \omega=\left.3 \Omega\right|_{\text {S }}$, где $\Omega=\omega^{123}-\omega^{145}+\omega^{167}-\omega^{246}-\omega^{257}-$ $-\omega^{347}+\omega^{356}$. Найдем оператор Хитчина [10] $K_{d \omega}$ для сферы $\mathbf{S}^{3,3}$. Рассмотрим сначала точку $x=e_{4} \in \mathbf{S}^{3,3}$. Касательное пространство $T_{x} \mathbf{S}^{3,3}$ имеет ортонормированный базис и форму объема $\mu=\omega^{123567} .2$-форма $d \omega$ на $T_{x} \mathbf{S}^{3,3}$ имеет вид

$$
d \omega_{x}=3\left(\omega^{123}+\omega^{167}-\omega^{257}+\omega^{356}\right) .
$$

Вследствие инвариантности формы $d \omega_{x}$ относительно подгруппы изотропии $S L(3, \mathbf{R}) \subset G_{2}{ }^{*}$, действующей на $T_{x} \mathbf{S}^{3,3}$, достаточно вычислить $K_{d \omega}$ на одном вектоpe, например $K_{d \omega}\left(\boldsymbol{e}_{1}\right)$ :

$$
\begin{aligned}
& \mathrm{t}_{e_{1}} d \omega_{x} \wedge d \omega_{x}=9\left(\omega^{23}+\omega^{67}\right)\left(\omega^{123}+\omega^{167}-\omega^{257}+\omega^{356}\right)= \\
& =9\left(\omega^{12367}+\omega^{12367}\right)=18 \omega^{12367}=-18 \mathrm{e}_{e_{5}} \omega^{123567}=-18 \mathrm{e}_{e_{5}} \mu
\end{aligned}
$$

Поэтому $K_{d \omega}\left(\boldsymbol{e}_{1}\right)=-18 \boldsymbol{e}_{5}=18 \boldsymbol{e}_{4} \times \boldsymbol{e}_{1}=18 P\left(\boldsymbol{e}_{1}\right)$. Отсюда следует, что $I_{d \omega}=P_{x}$. Из инвариантности почти пара-комплексной структуры Кэли $P$ и формы $\Omega$ относительно группы $G_{2}{ }^{*}$, действующей транзитивно на $\mathbf{S}^{3,3}$, получаем, что равенство $I_{d \omega}=P_{x}$ имеет место во всех точках псевдосферы $\mathbf{S}^{3,3}$.

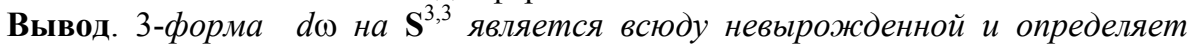
почти пара-комплексную структуру $I_{d \omega}$ на $\mathbf{S}^{3,3}$, которая совпадает с почти паракомплексной структурой Кэли Р. 
Вычислим тензор Нейенхейса [9] почти пара-комплексной структуры $P$ :

$$
N_{P}(X, Y)=2([X, Y]+[P X, P Y]-P[P X, Y]-P[X, P Y]) .
$$

Для этого найдем ковариантную производную тензора $P$. Поскольку $\boldsymbol{n}(x)=x$, то для любого касательного вектора $X \in T_{x} \mathbf{S}^{3,3}$ имеем $D \boldsymbol{n}_{x}(X)=X$. Будем считать, что касательные векторы $X, Y \in T_{x} \mathbf{S}^{3,3}$ продолжены на пространство $\mathbf{R}^{7}$ как постоянные векторные поля и $\boldsymbol{n}(x)=x$ также определено на $\mathbf{R}^{7} \backslash\{0\}$.

Тогда имеют место равенства $\left(\nabla_{X} P\right) Y=\operatorname{pr}_{x}\left(D_{X}(P Y)\right)=\operatorname{pr}_{x}\left(D_{X}(\boldsymbol{n} \times Y)\right)=\operatorname{pr}_{x}(X \times Y)$, где $\operatorname{pr}_{x}(Z)=Z+g(Z, \boldsymbol{n}) \boldsymbol{n}$ - ортогональная в $\mathbf{R}^{3,4}$ проекция на касательное пространство $T_{x} \mathbf{S}^{3,3}$, здесь мы учитываем, что $g(\boldsymbol{n}, \boldsymbol{n})=-1$. Получаем

$$
\left(\nabla_{X} P\right) Y=X \times Y+g(\boldsymbol{n}, X \times Y) \boldsymbol{n}=X \times Y+\omega(X, Y) \boldsymbol{n} .
$$

Замечание. Из полученного выражения $\nabla_{X} P$ сразу вытекает кососимметричность оператора $\nabla_{X} P$ и, в частности, свойство $\nabla_{\mathrm{X}}(P) X=0$ приближенной паракэлеровости многообразия $\mathbf{S}^{3,3}$ и равенство $\nabla_{P X} P=-P \nabla_{X} P$.

Теорема 4. Тензор Нейенхейса $N_{P}(X, Y)$ почти пара-комплексной структуры Кэли Р на $\mathbf{S}^{3,3}$ имеет вид

$$
N_{P}(X, Y)=-8 \boldsymbol{n} \times(X \times Y) .
$$

Доказательство. Прямое вычисление с использованием формулы $\left(\nabla_{X} P\right) Y=X \times Y+\omega(X, Y) \boldsymbol{n}$ и равенства $(\boldsymbol{n} \times X) \times Y=-\boldsymbol{n} \times(X \times Y)-g(X, Y) \boldsymbol{n}$ для $X, Y \perp \boldsymbol{n}$ :

$$
\begin{gathered}
N_{P}(X, Y) / 2=[X, Y]+[P X, P Y]-P[P X, Y]-P[X, P Y]= \\
=\nabla_{X}(Y)-\nabla_{\mathrm{Y}}(X)+\nabla_{\mathrm{PX}}(P Y)-\nabla_{\mathrm{PY}}(P X)-P \nabla_{\mathrm{PX}}(Y)+P \nabla_{\mathrm{Y}}(\mathrm{P} X)-P \nabla_{\mathrm{X}}(P Y)+P \nabla_{\mathrm{PY}}(X)= \\
=\nabla_{\mathrm{PX}}(P) Y-\nabla_{\mathrm{PY}}(P) X+P \nabla_{\mathrm{Y}}(P) X-P \nabla_{\mathrm{X}}(P) Y= \\
=(P X) \times Y+\omega(P X, Y) \boldsymbol{n}-((P Y) \times X+\omega(P Y, X) \boldsymbol{n})+P(Y \times X+\omega(Y, X) \boldsymbol{n})-P(X \times Y+\omega(X, Y) \boldsymbol{n})= \\
=(\boldsymbol{n} \times X) \times Y-(\boldsymbol{n} \times Y) \times X+\boldsymbol{n} \times(Y \times X)-\boldsymbol{n} \times(X \times Y)=-4 \boldsymbol{n} \times(X \times Y)
\end{gathered}
$$

Теорема 5. Фундаментальная 2-форма $\omega$ почти пара-комплексной структурь Кэли на $\mathbf{S}^{3,3}$ является собственной для оператора Лапласа: $\Delta \omega=-12 \omega$.

Доказательство. Полностью повторяет доказательство теоремы 3 с одним отличием: $\delta=+* d *$. Действительно, на $k$-формах кодифференциал $\delta$ имеет выражение $\delta=(-1)^{k} *^{-1} d *=(-1)^{k n+n+1+\eta} * d *$, где $\eta$ - число «минусов» метрического тензоpa $g$. В нашем случае $\eta=3$ и $n=6$. Следовательно, $\delta=+* d *$.

Пара-комплексная структура на $\mathbf{S}^{\mathbf{3 , 3}}(\boldsymbol{i})$. На псевдосфере $\mathbf{S}^{3,3}$ существуют интегрируемые пара-комплексные структуры. Это следует из того, что $\mathbf{S}^{3,3}$ диффеоморфно прямому произведению трехмерных многообразий. Рассмотрим стереографическую проекцию $\mathbf{S}^{3,3}$ из точки начала координат $\mathbf{R}^{7}$ на часть цилиндра $D^{3} \times \mathbf{S}^{3}$, где $\mathbf{S}^{3}$ - стандартная единичная сфера в пространстве $\mathbf{R}^{4}$ с координатами $\left(x_{4}, x_{5}, x_{6}, x_{7}\right)$ и $D^{3}$ - единичный шар в пространстве $\mathbf{R}^{3}$ с координатами $\left(x_{1}, x_{2}, x_{3}\right)$, $x_{1}^{2}+x_{2}^{2}+x_{3}^{2}<1$. Прямая, проходящая через начало координат и точку $x=\left(x_{1}\right.$, $\left.x_{2}, \ldots, x_{7}\right)$ псевдосферы $-x_{1}^{2}-x_{2}^{2}-x_{3}^{2}+x_{4}^{2}+x_{5}^{2}+x_{6}^{2}+x_{7}^{2}=1$, пресекает цилиндр $D^{3} \times \mathbf{S}^{3}$ в точке $y$ с координатами

$$
y_{i}=\frac{x_{i}}{\sqrt{x_{4}^{2}+x_{5}^{2}+x_{6}^{2}+x_{7}^{2}}}, \quad i=1,2, \ldots, 7 .
$$

Ясно, что последние переменные $\left(y_{4}, y_{5}, y_{6}, y_{7}\right)$ описывают трехмерную сферу $\mathbf{S}^{3}$. Остальные координаты меняются в единичном шаре $D^{3}$ в пространстве $\mathbf{R}^{3}$. Дейст- 
вительно, пусть $\rho^{2}=x_{4}^{2}+x_{5}^{2}+x_{6}^{2}+x_{7}^{2}$. На псевдосфере $\rho^{2} \geq 1$. Тогда из уравнения псевдосферы следует, что

$$
\frac{x_{1}^{2}+x_{2}^{2}+x_{3}^{2}}{x_{4}^{2}+x_{5}^{2}+x_{6}^{2}+x_{7}^{2}}=1-\frac{1}{x_{4}^{2}+x_{5}^{2}+x_{6}^{2}+x_{7}^{2}} \text { или } 0 \leq y_{1}^{2}+y_{2}^{2}+y_{3}^{2}=1-\frac{1}{\rho^{2}}<1 \text {. }
$$

Через каждую точку $\left(y_{1}, y_{2}, y_{3}\right) \in D^{3}$, при фиксированной точке $\left(y_{4}, y_{5}, y_{6}, y_{7}\right) \in \mathbf{S}^{3}$, проходит единственная прямая, пересекающая псевдосферу в точке $x_{i}=\rho \cdot y_{i}, i=1,2, \ldots, 7$ при $\rho=1 / \sqrt{1-y_{1}^{2}-y_{2}^{2}-y_{3}^{2}}$ и, наоборот, каждая прямая, проходящая через начало координат и точку $x \in \mathbf{S}^{3,3}$, пересекает шар $D^{3}$ при определенных $\left(y_{1}, y_{2}, y_{3}\right)$.

Поскольку прямое произведение $D^{3} \times \mathbf{S}^{3}$ естественно допускает паракомплексные структуры, то диффеоморфизм стереографической проекции $f: \mathbf{S}^{3,3}$ $\rightarrow D^{3} \times \mathbf{S}^{3}$ позволяет перенести пара-комплексные структуры с прямого произведения трехмерных многообразий $D^{3} \times \mathbf{S}^{3}$ на сферу $\mathbf{S}^{3,3}$. Отметим, что по построению, $D^{3} \times \mathbf{S}^{3}$ является псевдоримановым сигнатуры $(3,3)$.

\section{6. Почти комплексная и почти пара-комплексная структуры на алгебре Кэли}

Пространство $\mathbf{R}^{7}=\mathbf{R}^{3,4}$ чисто мнимых чисел $X=x_{1} \boldsymbol{e}_{1}+x_{2} \boldsymbol{e}_{2}+\ldots+x_{7} \boldsymbol{e}_{7}$ разбивается конусом $N_{0}(X)=x_{1}^{2}+x_{2}^{2}+x_{3}^{2}-x_{4}^{2}-\cdots-x_{7}^{2}=0$ на два открытых множества $\mathbf{R}^{7+}$ и $\mathbf{R}^{7-}$ элементов, для которых $N_{0}(X)>0$ и $N_{0}(X)<0$ соответственно. Рассмотрим прямые произведения этих множеств с вещественной прямой $\mathbf{R}_{0}$ алгебры Кэли:

$$
\mathbf{R}^{8+}=\mathbf{R}_{0} \times \mathbf{R}^{7+} \text { и } \quad \mathbf{R}^{8-}=\mathbf{R}_{0} \times \mathbf{R}^{7-} .
$$

В данном разделе мы определим неинтегрируемую почти комплексную структуру на открытом множестве $\mathbf{R}^{8+}$ и неинтегрируемую пара-комплексную структуру на $\mathbf{R}^{8-}$.

Почти комплексная структура Кэли. Рассмотрим пространство $\mathbf{R}^{8+}=\mathbf{R}_{0} \times \mathbf{R}^{7+}$ и определим на нем неинтегрируемую почти комплексную структуру $J$. На пространстве $\mathbf{R}_{0}$ определено единичное векторное поле $n_{1}=\boldsymbol{e}_{0}=1$. Определим единичное векторное поле $n_{2}(u)$ на $\mathbf{R}^{8+}$ формулой $n_{2}(u)=U /\|U\|$, где $\|U\|=\sqrt{N_{0}(U)}>0, u=u_{0}+U \in \mathbf{R}^{8+}=\mathbf{R}_{0} \times \mathbf{R}^{7+}$. Отметим, что $\left\langle n_{2}, n_{2}\right\rangle=1$, a $n_{2} n_{2}=-1$. Определим оператор $J: \mathbf{R}^{8} \rightarrow \mathbf{R}^{8}$ в точке $u=u_{0}+U \in \mathbf{R}_{0} \times \mathbf{R}^{7+}$ формулой

$$
J(y)=n_{2}(u) \cdot y=\frac{U}{\|U\|} y .
$$

Поскольку $n_{2} n_{2}=-1$, то $J^{2}=-I d$ и получаем почти комплексную структуру в точке $u=u_{0}+U \in \mathbf{R}^{8+}$.

Фундаментальная форма $\omega(X, Y)=g(J X, Y)$, соответствующая $J$, легко вычисляется с учетом свойства $n_{2} \times Y=n_{2} Y+<n_{2}, Y>$ :

$$
\begin{gathered}
\omega(y, z)=\langle J(y), z\rangle=\left\langle n_{2} y, z\right\rangle=\left\langle n_{2}\left(y_{0}+Y\right), z_{0}+Z\right\rangle= \\
=y_{0}\left\langle n_{2}, z_{0}+Z\right\rangle+\left\langle n_{2} Y, z_{0}+Z\right\rangle=y_{0}\left\langle n_{2}, z\right\rangle+\left\langle n_{2} \times Y, Z\right\rangle-\left\langle n_{2}, y\right\rangle z_{0}= \\
=n_{1} \wedge n_{2}^{*}(y, z)+\left\langle n_{2} \times Y, Z\right\rangle .
\end{gathered}
$$


Также легко вычисляется производная $D_{x}(J)$ тензорного поля $J$ в направлении вектора $x=x_{0}+X \in \mathbf{R}^{8+}$. Поскольку пространство $\mathbf{R}^{8+}$ является открытым подмножеством в $\mathbf{R}^{8}$, то можно считать векторы $x, y$ с параллельными векторными полями на $\mathbf{R}^{8+}$ и тогда $D_{x}(J)(y)=D_{x}\left(n_{2}\right) y$.

Векторное поле $n_{2}(u)$ не зависит от переменной $u_{0}$, поэтому его производная в направлении вектора $x=\boldsymbol{e}_{0}$ равна нулю. Поэтому достаточно вычислить $D_{x}\left(n_{2}\right)$ только в направлении вектора $x=X \in \mathbf{R}^{7+}$. Эта производная находится простым дифференцированием единичного векторного поля $n_{2}(u)$. Если $u=u_{0}+U \in \mathbf{R}^{8+}$, то $n_{2}(u)=U /\|U\|$. Поэтому из формулы $D_{x}(J)(y)=D_{x}\left(n_{2}\right) y$ получаем следующее выражение для производной почти комплексной структуры Кэли $J$ на $\mathbf{R}^{8+}$ в точке $u=u_{0}+U$ в направлении вектора $x=x_{0}+X$ :

$$
D_{x}(J) y=\left(d_{X} n_{2}\right) y=\frac{1}{\|U\|}\left(X-\left\langle X, n_{2}(u)\right\rangle n_{2}(u)\right) \cdot y .
$$

Используя это выражение, можно легко вычислить (так же, как и в теореме 4) тензор Нейенхейса $N(x, y)$ для векторов $x, y$, ортогональных $n_{2}(u)$. Вычислим внешний дифференциал 2-формы $\omega$ в точке $u=u_{0}+U$ с использованием полученной формулы для $D_{x}(J)$ :

$$
\begin{gathered}
d \omega(x, y, z)=x \omega(y, z)+y \omega(z, x)+z \omega(x, y)=\left\langle D_{x}(J) y, z\right\rangle+\left\langle D_{y}(J) z, x\right\rangle+\left\langle D_{z}(J) x, y\right\rangle= \\
=\frac{1}{\|U\|}\left(3\langle X \times Y, Z\rangle-\left\langle X, n_{2}(u)\right\rangle \omega(Y, Z)-\left\langle Y, n_{2}(u)\right\rangle \omega(Z, X)-\left\langle Z, n_{2}(u)\right\rangle \omega(X, Y)\right)= \\
=\frac{1}{\|U\|}\left(3 \Omega(X, Y, Z)-\left(n_{2}^{*} \wedge \omega\right)(X, Y, Z)\right) .
\end{gathered}
$$

Почти пара-комплексная структура Кэли. Рассмотрим пространство $\mathbf{R}^{8-}$ $=\mathbf{R}_{0} \times \mathbf{R}^{7-}$ и определим неинтегрируемую почти пара-комплексную структуру $P$. На прямой $\mathbf{R}_{0}$ определено единичное векторное поле $n_{1}=\boldsymbol{e}_{0}=1$. Определим единичное векторное поле $n_{2}(u)$ на $\quad \mathbf{R}^{8-}$ формулой $n_{2}(u)=U /\|U\|$, где $\|U\|=\sqrt{-N_{0}(U)}>0, \quad u=u_{0}+U \in \mathbf{R}^{8-}=\mathbf{R}_{0} \times \mathbf{R}^{7-}$. Отметим, что $\left\langle n_{2}, n_{2}\right\rangle=-1 \quad$ и $n_{2} n_{2}=+1$. Определим оператор $P: \mathbf{R}^{8} \rightarrow \mathbf{R}^{8}$ в точке $u=u_{0}+U \in \mathbf{R}_{0} \times \mathbf{R}^{7-}$ такой же формулой

$$
P(y)=n_{2}(u) \cdot y=\frac{U}{\|U\|} y .
$$

Легко видеть, что $P^{2}=I d$. Поэтому мы получаем неинтегрируемую почти пара-комплексную структуру на $\mathbf{R}^{8-}$. Совершенно аналогично вычисляются $D_{x}(P)$, фундаментальная форма $\omega(X, Y)=g(P X, Y)$, соответствующая $P$, и ее внешний дифференциал в точке $u=u_{0}+U$. Они имеют точно такой же вид, как и в случае почти комплексной структуры на $\mathbf{R}^{8+}$.

\section{ЛИТЕРАТУРА}

1. Agricola I., Bazzoni G., Goertsches J., et al. On the history of the Hopf problem // Diff. Geom. and Appl. 2018. V. 57. P. 1-9. DOI: doi.org/10.1016/j.difgeo.2017.10.014 (arXiv: 1708.01068 [math.HO]).

2. Agricola I., Borówka A., Friedrich T. S6 and the geometry of nearly Kähler 6-manifolds // Diff. Geom. and Appl. 2018. V. 57. P. 75-86. DOI: 10.1016/j.difgeo.2017.10.007 (arXiv: 1707.08591 [math.DG], 2017, 12 pages). 
3. Смоленцев Н.К. О почти комплексных структурах на шестимерных произведениях сфер // Ученые записки Казанского государственного университета. 2009. Т. 151. Кн. 4. C. $116-135$.

4. Alekseevsky D.V., Kruglikov B.S., Winther H. Homogeneous almost complex structures in dimension 6 with semi-simple isotropy // Ann. Glob. Anal. Geom. 2014. V. 46. P. 361-387. DOI: doi.org/10.1007/s10455-014-9428-y ( arXiv:1401.8187v2 [math.DG]).

5. Жевлаков К., Слинько А., Шестаков И., Ширшов А. Кольца, близкие к ассоциативным. М.: Наука, 1978.

6. Yokota I. Exceptional Lie groups. arXiv:0902.0431v1 [math.DG] 2009. DOI: doi=10.1.1. 401.8095\&rep $=$ rep1\&type $=$ pdf

7. Yokota I. Non-compact Simple Lie Group $G_{2}{ }^{\prime}$ of Type $G_{2} / /$ J. Fac. Sci. Shinshu University. 1977. V. 12. No. 1. P. 45-52.

8. Gray A. Vector cross products on manifolds // Trans AMS. 1969. V. 141. P. 465-504. DOI: doi.org/10.1090/S0002-9947-1969-0243469-5.

9. Алексеевский Д.В., Медори К., Томассини А. Однородные пара-кэлеровы многообразия Эйнштейна // УМН. 2009. Т. 64. Вып. 1(385). С. 3-50. DOI: https://doi.org/10.4213/rm9262.

10. Hitchin N.J. The geometry of three-forms in six dimensions // J. Diff. Geom. 2000. V. 55. P. 547-576.

11. Кобаяси Ш., Номидзу К. Основы дифференциальной геометрии. Т. 1 и 2. М.: Наука, 1981.

Статья поступила 14.02.2018 г.

Smolentsev N.K.(2018) ON ALMOST (PARA)COMPLEX CAYLEY STRUCTURES ON SPHERES $\mathrm{S}^{2,4}$ AND $\mathrm{S}^{3,3}$. Vestnik Tomskogo gosudarstvennogo universiteta. Matematika $i$ mekhanika [Tomsk State University Journal of Mathematics and Mechanics]. 53. pp. 22-38

DOI $10.17223 / 19988621 / 53 / 3$

Keywords: Cayley algebra, split Cayley algebra, G2 group, split-octonions, vector product, almost complex structure, almost para-complex structure, six-dimensional pseudo-Riemannian spheres.

It is well known that almost complex structures exist on the six-dimensional sphere $\mathbf{S}^{6}$ but the question of the existence of complex (ie, integrable) structures has not been solved so far. The most known almost complex structure on the sphere $\mathbf{S}^{6}$ is the Cayley structure which is obtained by means of the vector product in the space $\mathbf{R}^{7}$ of the purely imaginary octaves of Cayley $\mathbf{C a}$. There is another, split Cayley algebra $\mathbf{C a}^{\prime}$, which has a pseudo-Euclidean scalar product of signature $(4,4)$. The space of purely imaginary split octonions is the pseudo-Euclidean space $\mathbf{R}^{3,4}$ with a vector product. In the space $\mathbf{R}^{3,4}$, there are two types of spheres: pseudospheres $\mathbf{S}^{2,4}$ of real radius and pseudo sphere $\mathbf{S}^{3,3}$ of imaginary radius. In this paper, we study the Cayley structures on these pseudo-Riemannian spheres. On the first sphere $\mathbf{S}^{2,4}$, the Cayley structure defines an orthogonal almost complex structure $J$; on the second sphere, $\mathbf{S}^{3,3}$, the Cayley structure defines an almost para-complex structure $P$. It is shown that $J$ and $P$ are nonintegrable. The main characteristics of the structures $J$ and $P$ are calculated: the Nijenhuis tensors, as well as fundamental forms and their differentials. It is shown that, in contrast to the usual Riemann sphere $\mathbf{S}^{6}$, there are (integrable) complex structures on $\mathbf{S}^{2,4}$ and para-complex structures on $\mathbf{S}^{3,3}$.

AMS Mathematical Subject Classification: 53C15: 53C50; 53C30; 53C25; 53C38

SMOLENTSEV Nikolay Konstantinovich (Dr. Sci. in Physics and Mathematics, professor of Fundamental Mathematics department of Kemerovo State University, Kemerovo, Russian Federation). E-mail: smolennk@yandex.ru 


\section{REFERENCES}

1. Agricola I., Bazzoni G., Goertsches J., Konstantis P., Rollenske S. (2018) On the history of the Hopf problem. Diff. Geom. and Appl. 57. pp. 1-9. DOI: doi.org/10.1016/j.difgeo. 2017.10.014 (arXiv:1708.01068 [math.HO]).

2. Agricola I., Borówka A., Friedrich T. (2018) S6 and the geometry of nearly Kähler 6manifolds. Diff. Geom. and Appl. 57. pp. 75-86. DOI: 10.1016/j.difgeo.2017.10.007 (arXiv: 1707.08591 [math.DG], 2017, 12 pages.).

3. Smolentsev N.K. (2009) O pochti kompleksnykh strukturakh na shestimernykh proizvedeniyakh sfer [On almost complex structures on six-dimensional products of spheres]. Scientific Notes of Kazan State University. 151(4). pp. 116-135.

4. Alekseevsky D.V., Kruglikov B.S., Winther H. (2014) Homogeneous almost complex structures in dimension 6 with semi-simple isotropy. Ann. Glob. Anal. Geom. 46. pp. 361-387. DOI: doi.org/10.1007/s10455-014-9428-y ( arXiv:1401.8187v2 [math.DG]).

5. Zhevlakov K., Slin'ko A., Shestakov I., Shirshov A. (1978) Kol'tsa, blizkie $k$ assotsiativnym [Rings close to associative]. Moscow: Nauka.

6. Yokota I. (2009) Exceptional Lie groups. arXiv:0902.0431v1 [math.DG]. doi: 10.1.1.401.8095\&rep=rep1\&type $=$ pdf.

7. Yokota I. Non-compact Simple Lie Group $G_{2}{ }^{\prime}$ of Type $G_{2}(1977)$ Jour. Fac. Sci. Shinshu University. 12(1). pp. 45-52.

8. Gray A. (1969) Vector cross products on manifolds. Trans. AMS. 141. pp. 465-504. DOI: doi.org/10.1090/S0002-9947-1969-0243469-5.

9. Alekseevsky D.V., Medori C., Tomassini A. (2009) Homogeneous para-Kähler Einstein manifolds. Russ. Math. Surv. 64(1). pp. 1-43. DOI: https://doi.org/10.1070/ RM2009v064n01ABEH004591.

10. Hitchin N.J. (2000) The geometry of three-forms in six dimensions. J. Diff. Geom. 55. pp. 547-576.

11. Kobayashi S., Nomizu K. (1963) Foundations of Differential Geometry. Vol. 1 and 2. New York, London: Interscience Publ. 